\title{
Mayer-Rokitansky-Kuster-Hauser Syndrome
}

National Cancer Institute

\section{Source}

National Cancer Institute. Mayer-Rokitansky-Kuster-Hauser Syndrome. NCI Thesaurus.

Code C124853.

A rare syndrome of unknown cause that occurs in females. It is characterized by underdeveloped or absent vagina and uterus in an otherwise phenotypically normal female with a normal 46,XX karyotype. Other abnormalities include unilateral renal agenesis, skeletal abnormalities, hearing loss, and heart defects. 